\title{
Perturbation During Treadmill Training Improves Dynamic Balance and Gait in Parkinson's Disease: A Single-Blind Randomized Controlled Pilot Trial
}

Neurorehabilitation and Neural Repair 2017, Vol. 31 (8) 758-768 (C) The Author(s) 2017 Reprints and permissions: sagepub.com/journalsPermissions.nav DOI: $10.1177 / 1545968317721976$ journals.sagepub.com/home/nnr $@$ SAGE

\author{
Simon Steib, PhD', Sarah Klamroth, PT, MA', Heiko Gaßner, PhD', \\ Cristian Pasluosta, PhD $^{1,3}$, Björn Eskofier, $\mathbf{P h D}^{\prime}$, Jürgen Winkler, $\mathbf{M D}^{2}$, \\ Jochen Klucken, $\mathbf{M D}^{2}$, and Klaus Pfeifer, PhD' $^{1}$
}

\begin{abstract}
Background. Gait and balance dysfunction are major symptoms in Parkinson's disease (PD). Treadmill training improves gait characteristics in this population but does not reflect the dynamic nature of controlling balance during ambulation in everyday life contexts. Objective. To evaluate whether postural perturbations during treadmill walking lead to superior effects on gait and balance performance compared with standard treadmill training. Methods. In this single-blind randomized controlled trial, 43 PD patients (Hoehn \& Yahr stage 1-3.5) were assigned to either an 8-week perturbed treadmill intervention $(n=21)$ or a control group $(n=22)$ training on the identical treadmill without perturbations. Patients were assessed at baseline, postintervention, and at 3 months' follow-up. Primary endpoints were overground gait speed and balance (Mini-BESTest). Secondary outcomes included fast gait speed, walking capacity (2-Minute Walk Test), dynamic balance (Timed Up-and-Go), static balance (postural sway), and balance confidence (Activities-Specific Balance Confidence $[A B C]$ scale). Results. There were no significant between-group differences in change over time for the primary outcomes. At postintervention, both groups demonstrated similar improvements in overground gait speed $(P=.009)$, and no changes in the Mini-BESTest $(P=.64 I)$. A significant group-by-time interaction $(P=.048)$ existed for the Timed Up-and-Go, with improved performance only in the perturbation group. In addition, the perturbation but not the control group significantly increased walking capacity $(P=.038)$. Intervention effects were not sustained at follow-up. Conclusions. Our primary findings suggest no superior effect of perturbation training on gait and balance in PD patients. However, some favorable trends existed for secondary gait and dynamic balance parameters, which should be investigated in future trials.
\end{abstract}

\section{Keywords}

exercise, rehabilitation, Parkinson's disease, balance, postural instability, mobility, physical activity, perturbation, treadmill

\section{Introduction}

Parkinson's disease (PD) is a progressive neurological disorder with a growing prevalence worldwide. ${ }^{1}$ It is characterized by motor impairments, most importantly, bradykinesia, rigidity, tremor, and postural instability. ${ }^{2,3}$ Gait and balance dysfunction are extremely common in this population, increase during the disease course, and lead to a subsequent loss of mobility and quality of life. ${ }^{4,5}$ Critically, they also contribute to the increased risk of falling in PD patients. , $^{\text {P }}$

Pharmacological treatment options for gait and balance impairments are limited. ${ }^{8}$ Consequently, there is an urgent need for nonpharmacological strategies, ${ }^{5}$ and rapidly growing evidence supports the crucial role of exercise therapy., ${ }^{9,10}$ In a recent meta-analysis, we demonstrated comprehensive evidence for the positive effects of exercise on balance function in PD. ${ }^{11}$ Additionally, treadmill training has been suggested to be particularly effective for gait rehabilitation in PD patients. In a recent Cochrane review, Mehrholz and

'Friedrich-Alexander-University (FAU) Erlangen-Nürnberg, Erlangen, Germany

${ }^{2}$ University Hospital Erlangen, FAU Erlangen-Nürnberg, Erlangen, Germany

${ }^{3}$ University of Freiburg, Freiburg, Germany

Supplementary material for this article is available on the Neurorehabilitation \& Neural Repair website along with the online version of this article.

\section{Corresponding Author:}

Simon Steib, PhD, Division of Exercise and Health, Institute of Sport Science and Sport, Friedrich-Alexander-University Erlangen-Nürnberg. Gebbertstrasse 123b, 91058 Erlangen, Germany.

Email: simon.steib@fau.de 
colleagues ${ }^{12}$ concluded that treadmill intervention improves quantitative gait performance, including walking speed and stride length. While gait and balance control are inherently interconnected, treadmill walking does not entirely reflect the typical demands of ambulation in natural environments, particularly with regard to the dynamic control of balance during walking.

Consequently, novel concepts of treadmill training have emerged, designed to mimic typical demands of ambulation in natural environments. Interventions include virtual environments, ${ }^{13,14}$ split-belt systems, ${ }^{15}$ obstacle crossing tasks, ${ }^{16}$ and perturbations by applying external forces to the body, ${ }^{17}$ or sudden mediolateral or anterior-posterior belt translations. ${ }^{18-21}$ While these systems are promising, they have not been systematically evaluated in PD patients.

We recently provided first evidence that patients in early to mid-stages of PD immediately adapt their gait patterns during a single session of perturbed treadmill walking, and that these adaptations also transfer to overground walking. ${ }^{22}$ We used a unique form of perturbation, which comprised small 3-dimensional tilting movements of the treadmill platform, thereby mimicking the challenge of coping with a permanent and often stochastic change of the environmental conditions during walking on natural grounds. While these results are promising, the magnitude and sustainability of adaptations, when practiced regularly for longer periods, is still unknown.

Thus, the aim of this proof-of-concept trial was to investigate the effects of an 8 -week perturbation treadmill intervention on impaired gait and balance performance in PD patients compared with conventional treadmill training. We hypothesized that both interventions would improve patients' overground gait speed, but that balance performance would only increase with perturbation training. We further hypothesized that these balance improvements would lead to additional benefits for patients' gait performance.

\section{Methods}

\section{Design}

This single-blind, randomized controlled trial was registered (trial registration ID: NCT01856244), and approved by the local ethics committee (reference number: 181 12B). Patients were recruited at the Movement Disorder Unit (MDU) of the University Hospital Erlangen, and data were collected from May 2014 to July 2016 at the Institute of Sport Science and Sport (ISS) and the MDU of the Friedrich Alexander University (FAU) of ErlangenNürnberg. Patients participated in 16 sessions of a structured treadmill intervention of 40 minutes, 2 times per week for 8 weeks. Participants were asked to continue with their regular therapies and physical activity regimes throughout the study period. Assessments were performed at baseline (T1), postintervention (T2), and after a 12-week follow-up period (T3).

\section{Participants}

Forty-three patients with PD were randomly allocated to either an experimental group (Hoehn \& Yahr stage (H\&Y) $2.6 \pm 0.5$, Unified Parkinson's Disease Rating Scale (UPDRS)-III $17.7 \pm 6.1$ ) receiving perturbation treadmill training or a control group (H\&Y $2.5 \pm 0.5$, UPDRS-III 20.4 \pm 8.2 ) receiving conventional treadmill training. We used a computer-generated block-randomization (block size of 6) stratified by H\&Y stage (H\&Y 1-2 and H\&Y 2.5-3.5). An independent person (SS) generated the random allocation sequence, and group assignment was concealed during eligibility screening at the MDU (allocation schedule kept at ISS study center). All participants gave written informed consent prior to participation. Patients were informed that they would receive 1 of 2 different forms of treadmill training. However, they were unaware of the difference between these 2 interventions and the research hypotheses.

Patients fulfilling all of the following criteria were included in the study: (1) Diagnosis of idiopathic PD (following the diagnostic criteria of the UK PDS Brain Bank), (2) H\&Y of 1 to 3.5, (3) UPDRS-III subscore "gait" and/ or "postural stability" $\geq 1$, and (4) ability to walk independently without an assistive device. Patients were excluded if (1) they were diagnosed with any neurological disease other than PD, (2) they suffered from any severe cardiovascular or orthopedic condition that would affect performing the assessments and/or intervention, (3) they were unable to follow instructions from the assessor and/or care provider due to cognitive impairment, or (4) a major change in medication existed during the training period. Participants were instructed to continue with their regular physical activity routine and current medication scheme during the study. Changes were tracked during the assessment visits.

\section{Intervention}

The prototypic treadmill device used in this study has been previously described ${ }^{22}$ (see details in Supplementary File 1). Briefly, a standard medical treadmill (Mercury, $\mathrm{h} / \mathrm{p} / \mathrm{cos}-$ mos medical $\mathrm{GmbH}$ ) equipped with handrails at each side was mounted on a tiltable platform construction (zebris Medical $\mathrm{GmbH}$ ). The construction contains three pneumatic actuators (lifting capacity: $30 \mathrm{~mm}$ ), placed in a triangle below the platform surface. They induce small and audible three-dimensional tilting movements to the treadmill. The resulting changes in the surface conditions apply perturbations to the patient's balance control during walking. Control subjects walked on the identical treadmill 
device but without perturbations. A safety harness secured participants during walking.

Prior to the first training, each participant received one familiarization session in order to accommodate to the treadmill walking. Both groups participated in a total of 16 training sessions, carried out twice weekly within 8 to maximally 9 weeks. Each session consisted of 30 minutes of treadmill walking in the allocated condition, with additional 5 minutes for warm-up and cool-down (nonperturbed treadmill walking in both groups), respectively. Participants were encouraged to walk without holding onto the handrails, or with as little support as possible (only one hand/ fingers touching). Treadmill speed was individually progressed throughout the intervention period according to the patients' level of self-perceived exertion (Borg 6-20 scale; target range 12-15) and difficulty (Likert scale from 1 "not difficult" to 7 "extremely difficult"; target range $\leq 5$ ). Trained and experienced physiotherapists supervised the exercise sessions. In order to reduce the influence of feedback and therapist behavior, the amount and nature of instructions and feedback were rigorously restricted. Specifically, therapists were allowed to give feedback only related to (1) step length ("Try to make long steps"), (2) heel strike ("Try to make the first contact with your heel while pulling up your toes"), (3) arm swing ("Try to move both of your arms in the same direction as the diagonal leg"), and (4) posture ("Try to maintain an upright position while looking straight ahead"). One of these 4 instructions was provided every 10 minutes of the training, consequently resulting in a maximum of 3 instructions during the 30-minute training.

\section{Data Collection}

Primary and secondary endpoints were collected at baseline (T1), immediately postintervention (T2), and after a 12-week follow-up period (T3). All measurements and exercises were carried out with patients stable on medication (no severe offphases). Experienced movement disorders specialists at the ISS and the MDU assessed the outcome parameters. All assessors were blinded to the patients' group allocation and not involved in the training sessions. Twelve participants in the perturbation group disclosed their group allocation during the visit $(T 1 n=4 ; T 2 n=8)$.

Baseline physical activity status (steps per day) was recorded using hip-worn accelerometers (Actigraph GT3X+, Pensacola, FL, USA). Patients wore the device during daytime for 7 consecutive days. Data were analyzed if a minimum of 4 valid days ( $>9$ hours wear time) was available.

Primary Outcomes. Self-selected comfortable overground gait speed was assessed from a moving start on a 6-m distance. We instructed patients to walk at their normal, preferred pace. Patients stood at a tape mark on the floor and were asked to walk toward a $10-\mathrm{m}$ distant stop mark. We timed gait from the middle 6-m segment of the walk to avoid initialization, acceleration, or deceleration effects. ${ }^{23}$ The average of 3 trials was recorded.

The Mini Balance Evaluation Systems Test (MiniBESTest) score was used to assess patients' balance performance. ${ }^{24}$ It consists of a 14 -item scale ( 0 -2 points/ task; maximum score $=28$ ), evaluating the patients' balance abilities during standing, walking, and transferring as well as under dual-task conditions.

Secondary Outcomes. Fast overground gait speed was assessed similar to the comfortable gait speed test, but asking patients to walk as fast as safely possible. Three trials were recorded and averaged. The 2-Minute Walk Test ${ }^{25}$ was used to measure the patients' walking capacity. Patients walked up and down a 25-m long floor, turning around a cone at the end of each distance. They were instructed to walk as far as possible within 2 minutes, and no additional motivation was provided during the test. Treadmill gait speed during training was recorded to evaluate patients' performance change over time. The average training speed from the first and last 2 training sessions were used for analysis, respectively.

The Timed Up-and-Go (TUG) was used to evaluate the participants' balance during a complex dynamic task. ${ }^{26}$ Two trials were recorded and averaged. In addition, the MiniBESTest subscales were calculated to evaluate the function of different balance control systems: (1) anticipatory postural adjustments, (2) reactive postural control, (3) sensory orientation, and (4) dynamic balance. ${ }^{24}$ Static postural sway was assessed during 30 seconds of bipedal quiet standing (narrow stance) with eyes opened and eyes closed, respectively. Center of pressure (COP) excursions were collected at $100 \mathrm{~Hz}$ using a treadmill-integrated pressure sensor matrix $(108.4 \times 47.4 \mathrm{~cm}$, FDM-T, zebris Medical GmbH). COP velocity and COP root mean square (RMS) distance ${ }^{27}$ were calculated from the signal, and 3 trials were recorded and averaged. The Activities-Specific Balance Confidence (ABC) scale $^{28}$ was used to rate participants' balance self-confidence $(0=$ no confidence, $100=$ complete confidence $)$.

\section{Statistical Analysis}

No a priori sample size estimation was performed due to the pilot nature of this trial and the lack of data to estimate the additional effect of perturbation training. Post hoc power calculations were performed using the software $G^{*}$ Power (version 3.1.9.2). Homogeneity of groups in demographic and baseline variables were observed using independent-samples $t$-test for interval variables, chisquare test for categorical data, and Mann-Whitney $U$ test for ordinal variables. 


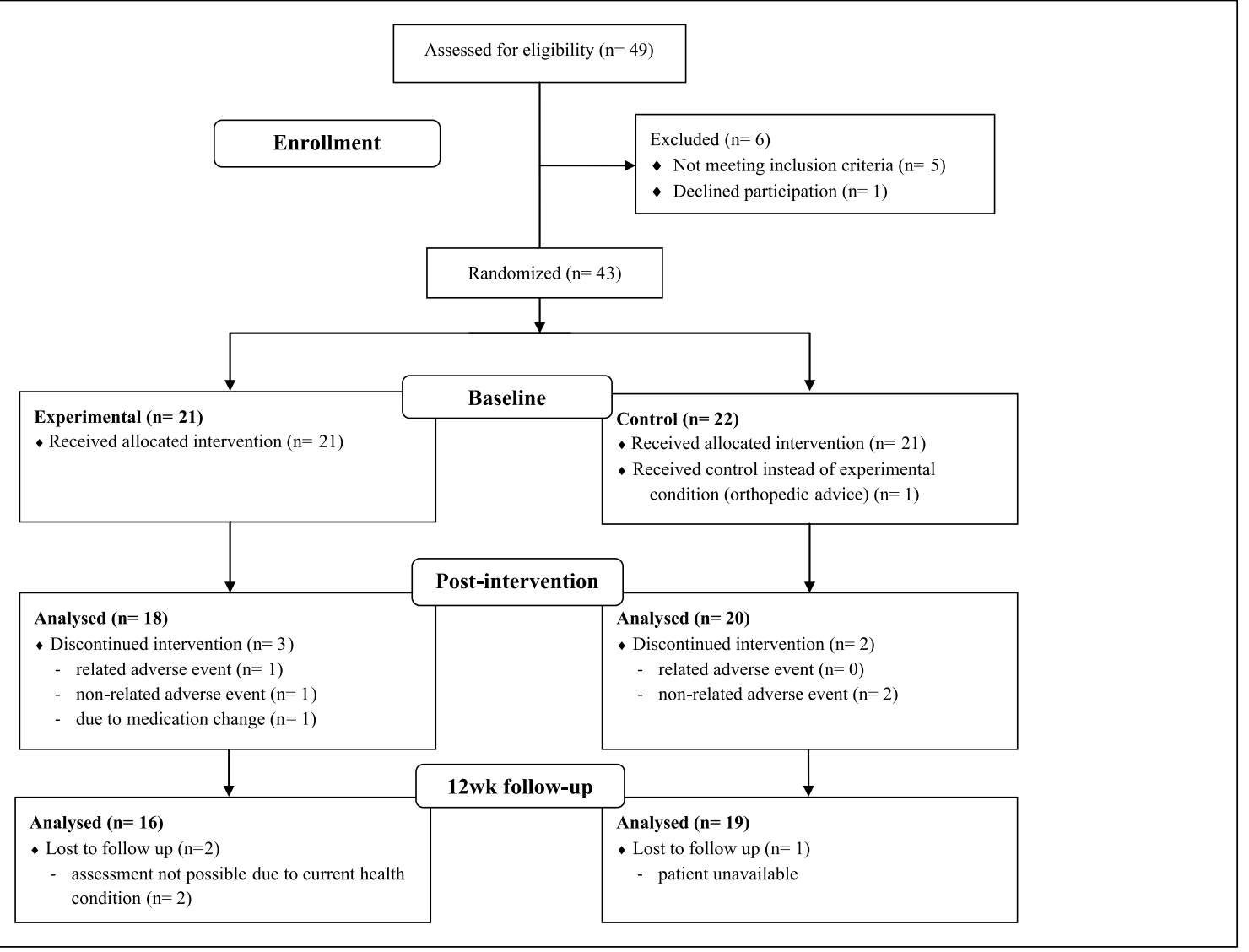

Figure I. Participants' flow (per protocol).

Normality of data was evaluated with the Shapiro-Wilk test. Levene's test was used to test for equality of variances. In case of normal distribution and variance homogeneity, parametric methods were used for analysis (overground and treadmill gait speed, 2-Minute Walk Test). A mixed repeated-measures analysis of variance (ANOVA) was implemented to test for Group $\times$ Time interaction effects (between-group differences in change over time). In addition, main effects of Group (perturbation vs control), and Time (within-subject change: T1 to T2 and T1 to T3) were analyzed. An analysis of covariance (ANCOVA) was further used to examine Group $\times$ Time interactions adjusted for baseline values. Since statistical effects were comparable, only results from the mixed ANOVA are presented.

Ordinal data and variables with skewed data distributions (Mini-BESTest, TUG, COP sway, ABC-scale) were analyzed using nonparametric methods. Change scores (T2 - T1 and T3 - T1) were calculated to test for Group $\times$ Time interactions using the Mann-Whitney $U$ test. Withingroup changes over time were evaluated with the Wilcoxon signed-rank test. Exploratory post hoc analyses examined changes over time in each group separately using paired $t$-tests (parametric) or Wilcoxon signed-rank test (nonparametric).
Statistical analyses included only those participants who completed the intervention and had complete data sets for the respective comparison (per protocol). Analysis of primary outcomes was adjusted for multiple testing using Bonferroni correction. Consequently, the $\alpha$-level was set at $P \leq .025$, and $P \leq .05$ for all secondary outcomes. Effect sizes for withingroup effects were computed using Cohen's $d$. Values between 0.2 and 0.49 were considered a small, between 0.5 and 0.79 a moderate, and of 0.8 and higher a large effect. All statistical analyses were performed using IBM SPSS Statistics version 23.0 (IBM Corp, Released 2015; Armonk, NY).

\section{Results}

Figure 1 presents the participants' flow. Out of 279 patients presented to the MDU during the study period, 49 patients were considered eligible and underwent further screening. From the 43 ultimately included patients, 5 (11.6\%; perturbation, $\mathrm{n}=3$; control, $\mathrm{n}=2$ ) dropped out during the intervention phase. In the perturbation group, 2 patients had to decline due to health reasons (ie, medication change; injurious fall at night). A third patient reported an increase of preexisting back pain during training, preventing further participation. In the control group, 2 patients dropped out due to medical 
Table I. Participants' Characteristics.

\begin{tabular}{|c|c|c|c|}
\hline & $\begin{array}{l}\text { Perturbation Group } \\
(\mathrm{n}=18), \text { Mean } \pm S D\end{array}$ & $\begin{array}{c}\text { Control Group } \\
(n=20), \text { Mean } \pm \text { SD }\end{array}$ & $P$ \\
\hline Age, years & $67.6 \pm 8.2$ & $62.5 \pm 7.9$ & .055 \\
\hline Gender, male/female, $n / n$ & $1 \mathrm{I} / 7$ & $16 / 4$ & $.200^{\mathrm{a}}$ \\
\hline Height, cm & $174.6 \pm 8.8$ & $175.8 \pm 8.6$ & .663 \\
\hline Weight, kg & $75.3 \pm 10.1$ & $79.2 \pm 9.0$ & .217 \\
\hline $\mathrm{BMI}, \mathrm{kg} / \mathrm{m}^{2}$ & $24.6 \pm 1.9$ & $25.6 \pm 2.3$ & .156 \\
\hline Disease duration, years & $7.9 \pm 4.0$ & $7.3 \pm 4.4$ & .644 \\
\hline LEDD, mg/d & $630.4 \pm 331.1$ & $645.7 \pm 280.8$ & .879 \\
\hline H\&Y stage & $2.6 \pm 0.5$ & $2.5 \pm 0.5$ & $.492^{\mathrm{a}}$ \\
\hline $\mathrm{H} \& \mathrm{Y} \mathrm{I}-2, \mathrm{n}$ & 3 & 5 & \\
\hline $\mathrm{H} \& \mathrm{Y} 2.5-3.5, \mathrm{n}$ & 15 & 14 & \\
\hline UPDRS-III & $17.7 \pm 6.1$ & $20.4 \pm 8.2$ & .257 \\
\hline Treadmill experience, $n$ & 9 & 9 & $.873^{\mathrm{a}}$ \\
\hline Gait speed, $\mathrm{m} / \mathrm{s}$ & $1.4 \pm 0.2$ & $1.4 \pm 0.2$ & .740 \\
\hline Gait speed fast, $\mathrm{m} / \mathrm{s}$ & $1.7 \pm 0.3$ & $1.8 \pm 0.3$ & .406 \\
\hline 2MWT, m & $188.4 \pm 27.1$ & $190.6 \pm 28.4$ & $.806^{\mathrm{b}}$ \\
\hline TUG, s & $9.9 \pm 2.6$ & $9.1 \pm 1.4$ & $.331^{\mathrm{b}}$ \\
\hline Mini-BESTest score, 0-28 & $24.1 \pm 3.3$ & $25.1 \pm 2.6$ & $.377^{\mathrm{b}}$ \\
\hline COP velocity eyes opened, $\mathrm{mm} / \mathrm{s}$ & $12.7 \pm 7.6$ & $13.2 \pm 5.9$ & .775 \\
\hline COP RMS eyes opened, $\mathrm{mm}$ & $7.5 \pm 1.9$ & $9.0 \pm 2.9$ & .613 \\
\hline COP velocity eyes closed, $\mathrm{mm} / \mathrm{s}$ & $24.9 \pm 17.8$ & $26.3 \pm 13.5$ & .424 \\
\hline COP RMS eyes closed, mm & $11.3 \pm 4.4$ & $12.5 \pm 4.2$ & .425 \\
\hline \multicolumn{4}{|l|}{ Physical activity level ${ }^{c}$} \\
\hline Steps per day & $5539 \pm 2162$ & $6147 \pm 2607$ & .468 \\
\hline
\end{tabular}

Abbreviations: BMI, body mass index; LEDD, levodopa equivalent daily dose; H\&Y, Hoehn \& Yahr stage; UPDRS, Unified Parkinson's Disease Rating Scale; 2MWT, 2-Minute Walk Test; TUG, Timed Up-and-Go; Mini-BESTest, Mini Balance Evaluation Systems Test; COP, center of pressure; RMS, root mean square.

${ }^{\mathrm{a}}$ Chi-square test.

bMann-Whitney $U$ test.

${ }^{c}$ Data from $n=16$ (perturbation) and $n=18$ (control).

conditions (ie, diagnosed leukemia, transient ischemic attack at home). In those participants who completed the intervention, compliance was high, with an average participation of $98.0 \%$ (minimum: $75 \%$ ) of all training sessions.

\section{Baseline Data}

Participants' baseline characteristics are presented in Table 1. No differences existed between groups for any of the observed demographic data or gait and balance variables. Eighteen patients (9 in each group) reported that they had already walked on a treadmill before participating in the study.

\section{Gait Outcomes}

Table 2 presents changes from baseline to postintervention. There were no significant between-group interaction effects for changes over time in any of the gait variables observed.

Both groups demonstrated significant improvements in comfortable gait speed from baseline to postintervention $(P=.009$; perturbation, $d=0.28$; control, $d=0.23)$, with no difference in change over time between groups $(P=.919)$. The perturbation group demonstrated a small positive effect on fast gait speed ( $d=0.35, P=.067)$, and a small significant improvement in the 2-minute walk $(d=0.23, P=.038)$, while no changes existed in the control group. However, between-group interaction effects were not statistically significant. Both groups demonstrated large improvements in treadmill gait speed over the intervention period $(P<.000$; perturbation, $d=0.95$; control, $d=0.85)$, which did not differ significantly between groups $(P=.959)$ (Figure 2$)$.

At follow-up (Table 3), the improvements in comfortable walking speed were not retained in both groups. Patients in the perturbation group still demonstrated increased performance in fast gait speed $(d=0.34)$ and the 2-minute walk $(d=0.20)$. However, these effects were not statistically different from baseline (Figure 2).

\section{Balance Outcomes}

There was no significant between-group difference in change over time for the primary balance outcome. 


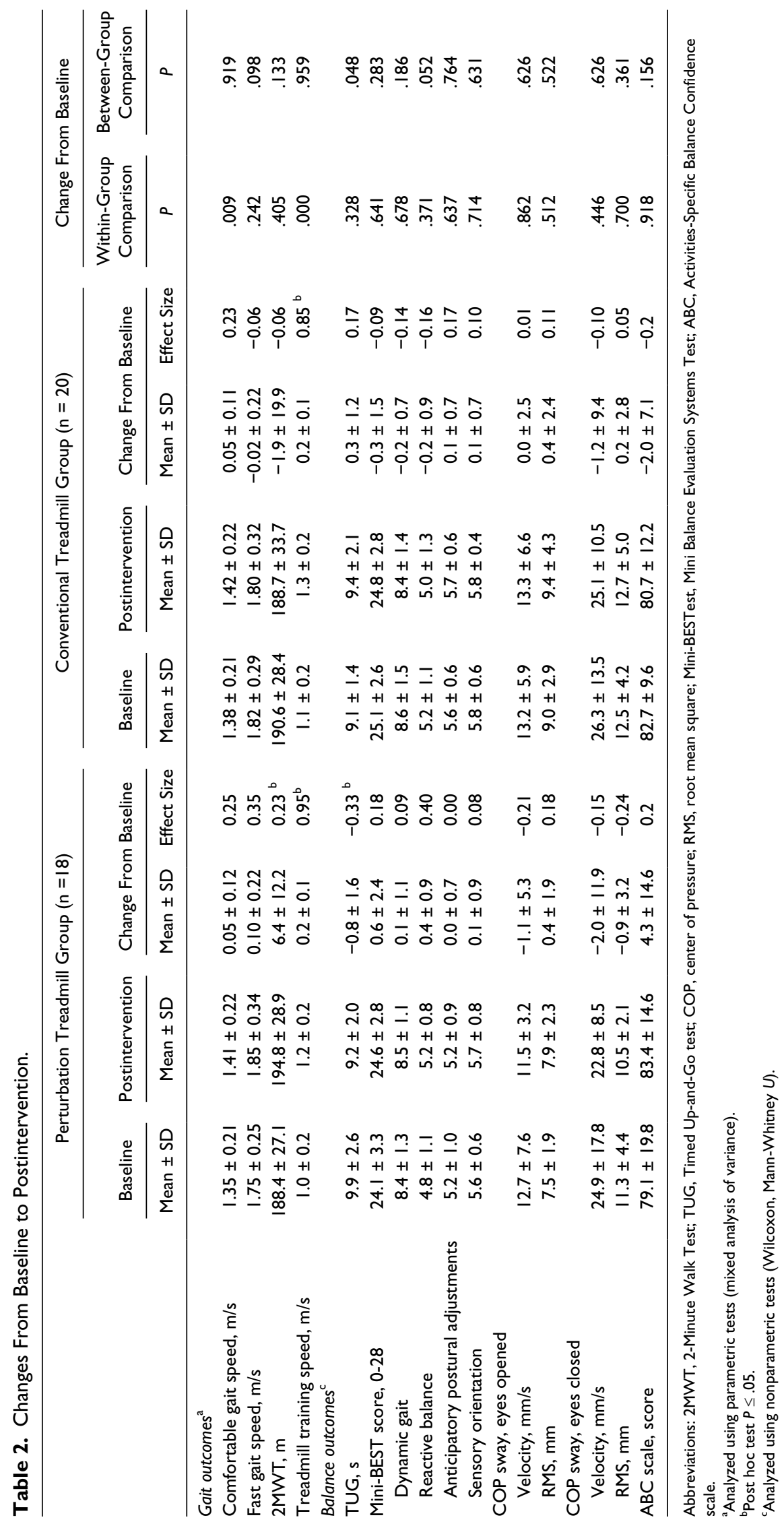




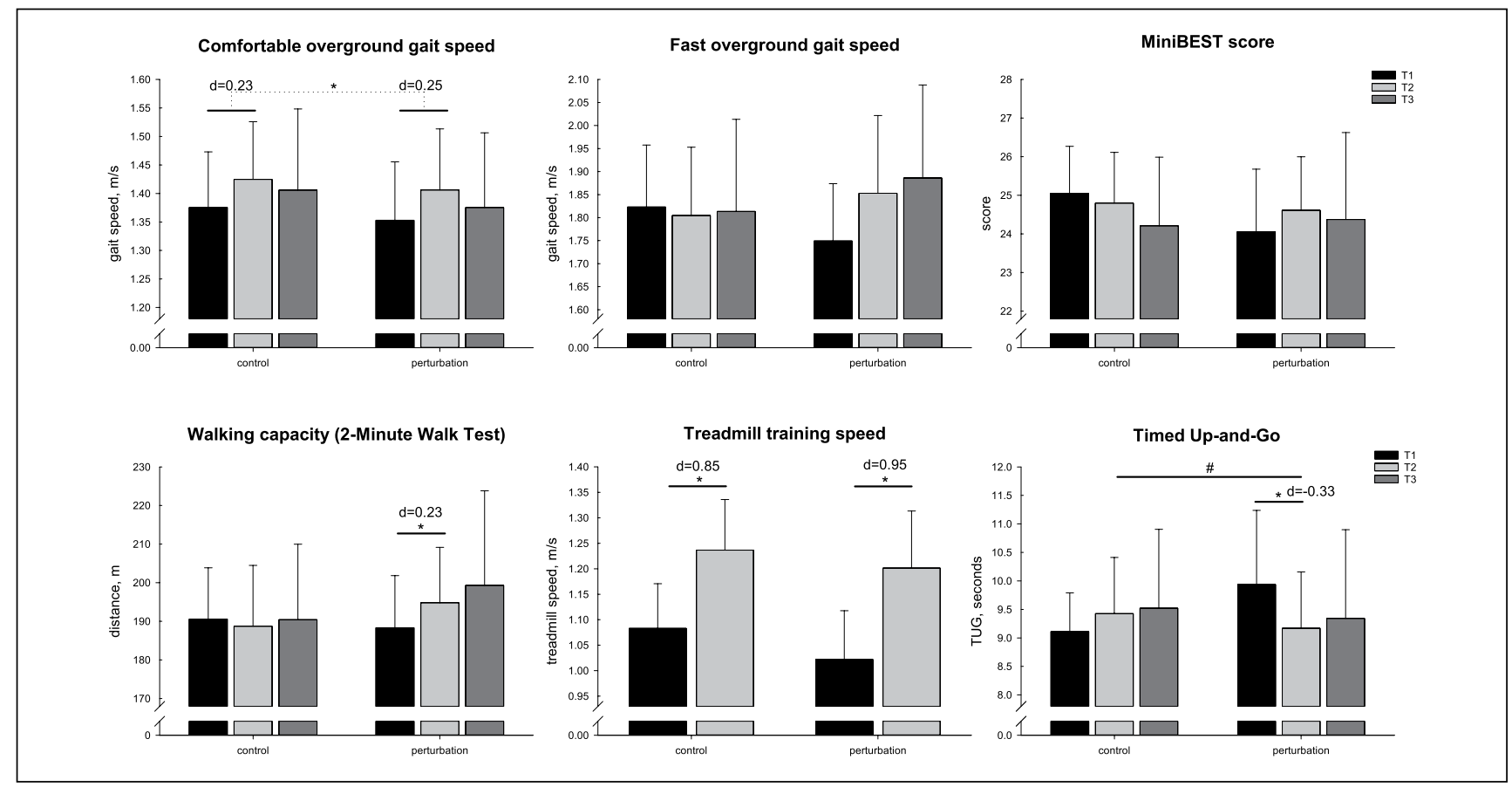

Figure 2. Gait and balance outcomes (mean, $95 \% \mathrm{Cl}$ ).

*Significant within-group change over time. \#Significant between-group difference in change over time (Group $\times$ Time interaction). Comfortable overground gait speed: dotted line indicates significant main effect for Time.

Performance in the Mini-BESTest score did not significantly change with intervention in both groups (Table 2). In the reactive postural control subscale, there was a trend toward benefits in the perturbation group but not the control group (between-group interaction, $P=.052$; perturbation, $d=0.40$; control, $d=-0.16$ ). A significant between-group interaction effect $(P=.048)$ existed for the TUG (Figure 2$)$, indicating that only the perturbation group improved TUG performance by reducing the time to complete the test from baseline to postintervention $(d=-0.33 ; P=.035)$. No effects were observed for COP sway (eyes opened and closed) or the ABC scale.

At follow-up (Table 3), no significant changes from baseline were observed except for COP sway. A small significant within-group effect existed for COP sway velocity in the eyes closed condition $(P=.030$; perturbation, $d=-0.18$; control, $d=-0.06)$, but the change over time did not differ between groups $(P=.233)$.

\section{Discussion}

The main results of this proof-of-concept trial do not indicate a superior effect of perturbation training. Both interventions led to increased overground gait speed of similar magnitude, and no improvement in balance performance. However, exploration of secondary outcomes indicated some interesting differences between treatments. Only those patients training with the perturbation stimulus improved TUG performance, and demonstrated a similar tendency in reactive postural control. This suggests differential adaptations in balance control which should be subject to further investigation. In addition, although no between-group interaction effect was detected, only the perturbation group significantly improved walking capacity in the 2-Minute Walk Test, while no effect was seen in the control group. The observed treatment effects in this short-term trial were mostly of small magnitude and not retained after 3 months' follow-up. The findings of this proof-of-concept study are based on a small sample of early to mid-stage PD patients with relatively high functional levels, which limit generalizability of conclusions.

\section{Effects on Gait}

Our results did not confirm that adding perturbations to treadmill walking would lead to larger effects on patients walking speed. Participants from both groups demonstrated improved comfortable gait speed from baseline to postintervention, which supports current evidence for the efficacy of treadmill training in PD. ${ }^{12}$ Since both treatments included intensive, repetitive gait training, an improvement in both groups was expected. However, contrary to what we hypothesized, perturbation training did not lead to larger improvements. Several reasons may explain this fact. First, baseline gait speed $(1.4 \pm 0.2 \mathrm{~m} / \mathrm{s})$ in our population was substantially higher compared to typical PD norms (0.9-1.2 


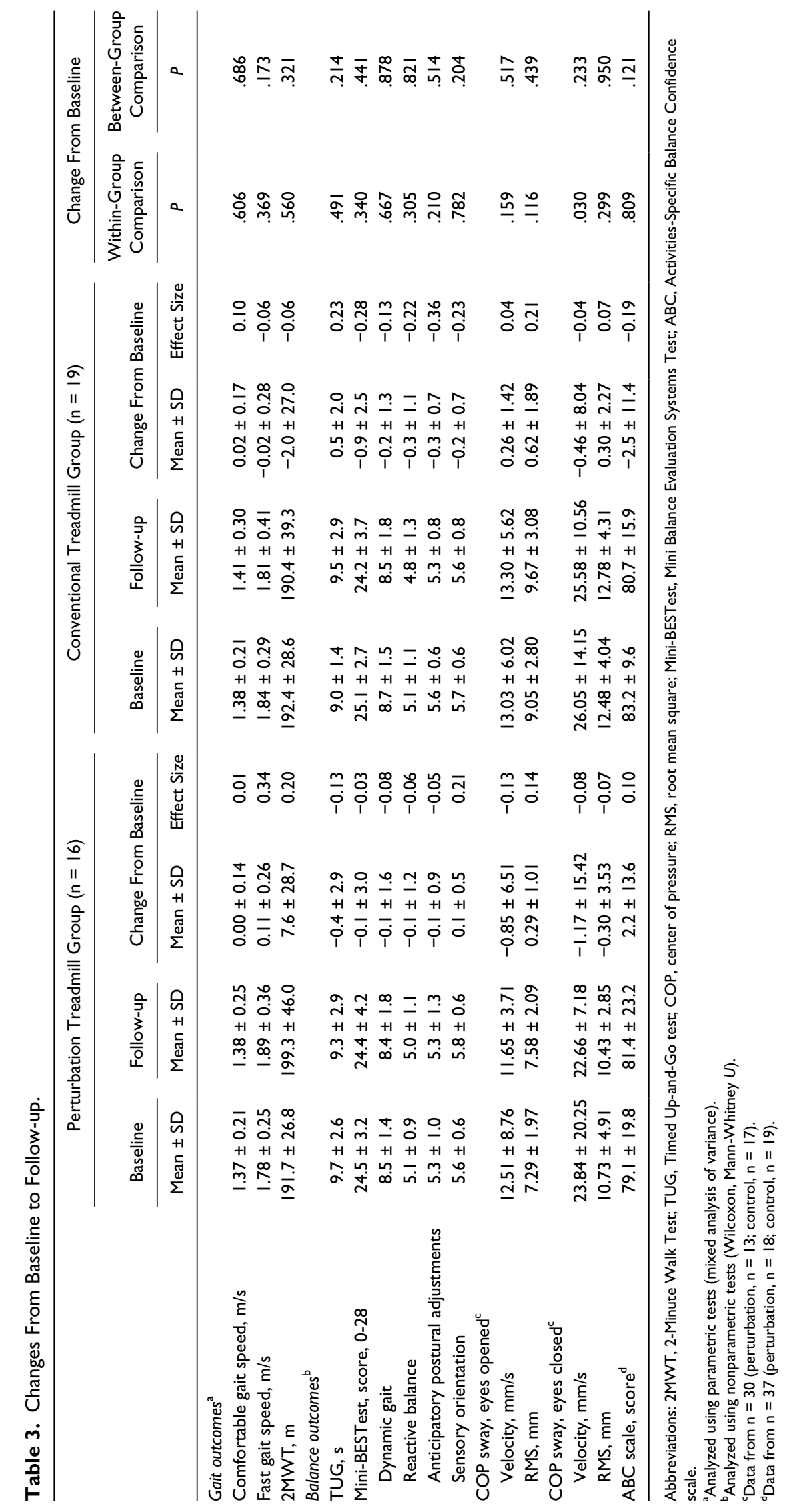


$\mathrm{m} / \mathrm{s})^{29,30}$ and comparable to healthy elderly. ${ }^{23}$ Thus, potential for improvement was rather small in most participants. A second reason might be that the perturbation stimulus targets qualitative aspects of gait, which are not reflected in gait quantity (speed). Consequently, parameters of gait variability or symmetry might be better suited to demonstrate the potential of perturbation training, particularly with regard to changes in postural control during walking. ${ }^{31} \mathrm{We}$ recently found a reduction in stride variability and symmetry after a single session of perturbed treadmill walking, which would support this concept. ${ }^{22}$

Between-group interaction effects on secondary gait outcomes confirmed that perturbation training had similar effects on walking performance compared to conventional treadmill training. It is however interesting to note that within-group changes revealed a small effect of perturbation training on walking capacity in the 2-Minute Walk Test immediately postintervention, which was not observed in the control group. The increased challenge during perturbed treadmill walking might have improved neuromuscular activation. This would result in a more economic gait, thereby lowering the energy costs during prolonged gait sequences. ${ }^{32}$ Second, one might also speculate that training with perturbations presents a more intense cardiorespiratory stimulus. However, we adjusted training intensity according to the patients' level of perceived exertion and difficulty, which resulted in comparable values between groups (mean exertion [6-20]: perturbation $=12.6$; control $=12.4$; mean difficulty [1-7]: perturbation $=3.3$, control $=3.4$ ).

\section{Effects on Balance}

Performance in the Mini-BESTest score did not significantly change in either of the groups with intervention. The results from static COP sway analysis point in a similar direction. This is contrary to findings from previous studies, demonstrating positive effects of unperturbed treadmill training on balance in PD patients. ${ }^{33,34}$ The high baseline test scores of our participants might explain this discrepancy. Analogous to gait outcomes, patients' Mini-BESTest performance at baseline was higher compared with typical ranges in mild to moderate PD. ${ }^{35,36}$ With an average of 24.6 $( \pm 2.9)$ out of 28 points, and 17 participants scoring $\geq 26$, from which 8 had the highest possible score, a considerable ceiling effect is likely.

Examination of secondary balance outcomes revealed that patients in the perturbation group, but not the control group, significantly improved in the TUG at postintervention. This is important, since the TUG requires balance control in multiple dynamic tasks, including initiation and termination of gait, transferring, and turning. Thus, this finding suggests that perturbed treadmill walking stimulates specific adaptations in dynamic balance control during ambulation. It is likely that the constant challenge of the postural control system during walking triggered these adaptations. ${ }^{6,11,37}$ Results from the MiniBEST reactive balance subscale further support this idea, with a similar trend toward larger improvements in the perturbation group. Overall, results from the observed balance parameters indicate that perturbed treadmill training may be effective in improving specific aspects of patients' balance control (ie, dynamic and reactive control), which should be further investigated.

\section{Mechanisms of Adaptation and Sustainability of Effects}

A growing body of evidence suggests that exercise-induced motor improvements are related to mechanisms of neural plasticity, ${ }^{9,38-40}$ which might explain the observed improvements in the present trial. Intensive task repetition, practice variability, and contextual interference, ${ }^{39}$ as well as combining aerobic exercise and skill practice have been shown to particularly drive neural plasticity. ${ }^{40,41}$ Perturbed treadmill training specifically addresses these aspects, and the selective changes in balance control in the perturbation group might support this concept. Overall, effects were rather small and non-permanent. Training for 8 weeks, twice weekly might not have been sufficient to produce long-lasting effects, and more knowledge about the optimal dose of exercise to induce mechanisms of plasticity in neurorehabilitation is warranted. ${ }^{40}$

\section{Compliance, Practicability, and Adverse Events}

The intervention was generally well accepted in both groups and compliance was very high. Twenty-two patients (perturbation, $\mathrm{n}=11$; control, $\mathrm{n}=11$ ) were able to walk completely without handrail support throughout the entire study, another 8 (perturbation, $\mathrm{n}=3$; control, $\mathrm{n}=5$ ) participants were able to walk without support for more than $50 \%$ of training sessions. Only 1 patient in the perturbation group was unable to walk without handrails for the entire training period. We observed a substantial improvement in treadmill training speed in most patients ( 27 of 36 patients improved training speed by $10 \%-43 \%$ ), irrespective of group allocation. Thus, both treadmill programs appeared to have good practicability in mild to moderately impaired PD patients.

The dropouts registered in this study were mostly unrelated to the intervention. Those potentially related mainly concerned preexisting orthopedic problems. In the pilot phase of this trial, another participant cancelled perturbed training due to acute knee pain. In addition, typical registered complaints were lower extremity or back pain (perturbation, $\mathrm{n}=4$; control, $\mathrm{n}=8$ ), which had existed already before study entry and did not worsen with training progression. A careful medical screening with a focus on orthopedic and cardiovascular risk factors is indispensable before administering patients to perturbed treadmill training. 


\section{Limitations}

This study presents some limitations. First, the sample size of this proof-of-concept study was rather small, and we were not able to obtain data from all participants at followup. Considering the rigorous control group (standard treadmill training) and that effect sizes for several outcomes indicated clinically relevant changes with time, it is likely that a lack of power existed for some comparisons to show statistical significance. Post hoc calculation revealed a power of $\leq 24 \%$ for detecting a group-by-time interaction at postintervention in the primary endpoints, and of $\leq 65 \%$ for secondary outcomes. Second, although particular efforts were taken to blind assessors, 12 patients in the perturbation group unintendedly revealed their group allocation, which may potentially have biased our results. Finally, most participants in our trial were relatively physically active, participating in additional regular exercise programs and physiotherapy. This is a common problem in exercise studies including elderly participants. ${ }^{42,43}$ It could be one explanation for the high performance level of participants at baseline, and may have diminished the potential for improvements in some of the measured outcomes.

\section{Conclusions}

In summary, our primary outcomes did not reveal a superior effect of perturbation compared with standard treadmill training on gait and balance in this proof-of-concept trial. However, some favorable trends existed for secondary gait and dynamic balance parameters in the perturbation group. Postural perturbations during treadmill walking lead to improvements in mobility-related dynamic balance and walking capacity, which were not present in the control group. These effects might indicate a more secure ambulation and need further investigation. The type of training in this study is novel and was well accepted by PD patients. It is a first step toward developing training regimes with additional challenges to postural gait control. Technical developments should aim at exploring the optimal nature and intensity of perturbations to stimulate neural plasticity mechanisms. The effects demonstrated in this study were mainly of small magnitude and short termed. The effectiveness and sustainability of longer or more intensive training periods remain to be determined in future investigations.

\section{Authors' Note}

The study sponsors were not involved in the study design, the collection, analysis, and interpretation of data, in the writing of the manuscript, or decision to submit the manuscript for publication.

\section{Acknowledgments}

We would like to thank the therapists involved in this study, Marie-Kristin Dunker, Lyusyena Novokreshchenova, Surendar
Devan, and Hasan Tariq. Furthermore, we thank Dr. Zacharias Kohl, Dr. Franz Marxreiter, Dr. Johannes Schlachetzki, and Dr. Martin Regensburger for their support in recruitment of participants, and Dr. Sebastian Krinner for providing orthopedic advice during the study.

\section{Declaration of Conflicting Interests}

The authors declared no potential conflicts of interest with respect to the research, authorship, and/or publication of this article.

\section{Funding}

The authors disclosed receipt of the following financial support for the research, authorship, and/or publication of this article: The study was financed by the Emerging Fields Initiative of the Friedrich-Alexander-University Erlangen-Nürnberg (FAU, Germany) and the German Foundation Neurology (Deutsche Stiftung Neurologie).

\section{References}

1. Wirdefeldt K, Adami HO, Cole P, Trichopoulos D, Mandel J. Epidemiology and etiology of Parkinson's disease: a review of the evidence. Eur J Epidemiol. 2011;26(suppl 1):S1-S58.

2. Tolosa E, Wenning G, Poewe W. The diagnosis of Parkinson's disease. Lancet Neurol. 2006;5:75-86.

3. Lees AJ, Hardy J, Revesz T. Parkinson's disease. Lancet. 2009;373:2055-2066.

4. Maetzler W, Nieuwhof F, Hasmann SE, Bloem BR. Emerging therapies for gait disability and balance impairment: promises and pitfalls. Mov Disord. 2013;28:1576-1586.

5. van der Kolk NM, King LA. Effects of exercise on mobility in people with Parkinson's disease. Mov Disord. 2013;28: 1587-1596.

6. Canning CG, Paul SS, Nieuwboer A. Prevention of falls in Parkinson's disease: a review of fall risk factors and the role of physical interventions. Neurodegener Dis Manag. 2014;4:203-221.

7. van der Marck MA, Klok MP, Okun MS, Giladi N, Munneke M, Bloem BR. Consensus-based clinical practice recommendations for the examination and management of falls in patients with Parkinson's disease. Parkinsonism Relat Disord. 2014;20:360-369.

8. Vu TC, Nutt JG, Holford NH. Progression of motor and nonmotor features of Parkinson's disease and their response to treatment. Br J Clin Pharmacol. 2012;74:267-283.

9. Abbruzzese G, Marchese R, Avanzino L, Pelosin E. Rehabilitation for Parkinson's disease: current outlook and future challenges. Parkinsonism Relat Disord. 2016;22(suppl 1):S60-S64.

10. Tomlinson CL, Patel S, Meek C, et al. Physiotherapy versus placebo or no intervention in Parkinson's disease. Cochrane Database Syst Rev. 2013;(9):CD002817.

11. Klamroth S, Steib S, Devan S, Pfeifer K. Effects of exercise therapy on postural instability in Parkinson disease: a metaanalysis. J Neurol Phys Ther. 2016;40:3-14.

12. Mehrholz J, Kugler J, Storch A, Pohl M, Hirsch K, Elsner B. Treadmill training for patients with Parkinson's disease. Cochrane Database Syst Rev. 2015;(8):CD007830. 
13. Hak L, Houdijk H, Steenbrink F, et al. Stepping strategies for regulating gait adaptability and stability. $J$ Biomech. 2013;46:905-911.

14. Peruzzi A, Zarbo IR, Cereatti A, Della Croce U, Mirelman A. An innovative training program based on virtual reality and treadmill: effects on gait of persons with multiple sclerosis. Disabil Rehabil. 2017;39:1557-1563.

15. Fasano A, Schlenstedt C, Herzog J, et al. Split-belt locomotion in Parkinson's disease links asymmetry, dyscoordination and sequence effect. Gait Posture. 2016;48:6-12.

16. Potocanac Z, Smulders E, Pijnappels M, Verschueren S, Duysens J. Response inhibition and avoidance of virtual obstacles during gait in healthy young and older adults. Hum Mov Sci. 2015;39:27-40.

17. IJmker T, Lamoth CJ, Houdijk H, van der Woude LH, Beek PJ. Postural threat during walking: effects on energy cost and accompanying gait changes. J Neuroeng Rehabil. 2014;11:71.

18. Lurie JD, Zagaria AB, Pidgeon DM, Forman JL, Spratt KF. Pilot comparative effectiveness study of surface perturbation treadmill training to prevent falls in older adults. $B M C$ Geriatr. 2013;13:49.

19. Sessoms PH, Wyatt M, Grabiner M, et al. Method for evoking a trip-like response using a treadmill-based perturbation during locomotion. $J$ Biomech. 2014;47:277-280.

20. Shapiro A, Melzer I. Balance perturbation system to improve balance compensatory responses during walking in old persons. J Neuroeng Rehabil. 2010;7:32.

21. Beurskens R, Wilken JM, Dingwell JB. Dynamic stability of individuals with transtibial amputation walking in destabilizing environments. $J$ Biomech. 2014;47:1675-1681.

22. Klamroth S, Steib S, Gaßner H, et al. Immediate effects of perturbation treadmill training on gait and postural control in patients with Parkinson's disease. Gait Posture. 2016;50 102-108.

23. Bohannon RW, Andrews AW, Thomas MW. Walking speed: reference values and correlates for older adults. $J$ Orthop Sports Phys Ther. 1996;24:86-90.

24. Franchignoni F, Horak F, Godi M, Nardone A, Giordano A. Using psychometric techniques to improve the Balance Evaluation Systems Test: the mini-BESTest. J Rehabil Med. 2010;42:323-331.

25. Stewart D, Burns Jma, Dunn SG, Roberts M. The two-minute walking test: a sensitive index of mobility in the rehabilitation of elderly patients. Clin Rehabil. 1990;4:273-276.

26. Podsiadlo D, Richardson S. The timed "Up \& Go": a test of basic functional mobility for frail elderly persons. $J \mathrm{Am}$ Geriatr Soc. 1991;39:142-148.

27. Prieto TE, Myklebust JB, Hoffmann RG, Lovett EG, Myklebust BM. Measures of postural steadiness: differences between healthy young and elderly adults. IEEE Trans Biomed Eng. 1996;43:956-966. doi:10.1109/10.532130.

28. Franchignoni F, Giordano A, Ronconi G, Rabini A, Ferriero G. Rasch validation of the Activities-specific Balance Confidence
Scale and its short versions in patients with Parkinson's disease. J Rehabil Med. 2014;46:532-539.

29. Hass CJ, Malczak P, Nocera J, et al. Quantitative normative gait data in a large cohort of ambulatory persons with Parkinson's disease. PLoS One. 2012;7:e42337.

30. Hass CJ, Bishop M, Moscovich M, et al. Defining the clinically meaningful difference in gait speed in persons with Parkinson disease. J Neurol Phys Ther. 2014;38:233-238.

31. Lord S, Galna B, Rochester L. Moving forward on gait measurement: toward a more refined approach. Mov Disord. 2013;28:1534-1543.

32. Fernández-Del-Olmo MA, Sanchez JA, Bello O, et al. Treadmill training improves overground walking economy in Parkinson's disease: a randomized, controlled pilot study. Front Neurol. 2014;5:191.

33. Cakit BD, Saracoglu M, Genc H, Erdem HR, Inan L. The effects of incremental speed-dependent treadmill training on postural instability and fear of falling in Parkinson's disease. Clin Rehabil. 2007;21:698-705.

34. Ganesan M, Sathyaprabha TN, Gupta A, Pal PK. Effect of partial weight-supported treadmill gait training on balance in patients with Parkinson disease. PM R. 2013;6:22-33.

35. Duncan RP, Leddy AL, Cavanaugh JT, et al. Comparative utility of the BESTest, mini-BESTest, and brief-BESTest for predicting falls in individuals with Parkinson disease: a cohort study. Phys Ther. 2013;93:542-550.

36. Mak MK, Auyeung MM. The mini-BESTest can predict Parkinsonian recurrent fallers: a 6-month prospective study. J Rehabil Med. 2013;45:565-571.

37. Schoneburg B, Mancini M, Horak F, Nutt JG. Framework for understanding balance dysfunction in Parkinson's disease. Mov Disord. 2013;28:1474-1482.

38. Jakowec MW, Wang Z, Holschneider D, Beeler J, Petzinger GM. Engaging cognitive circuits to promote motor recovery in degenerative disorders. Exercise as a learning modality. $J$ Hum Kinet. 2016;52:35-51.

39. Kitago T, Krakauer JW. Motor learning principles for neurorehabilitation. Handb Clin Neurol. 2013;110:93-103.

40. Petzinger GM, Fisher BE, McEwen S, Beeler JA, Walsh JP, Jakowec MW. Exercise-enhanced neuroplasticity targeting motor and cognitive circuitry in Parkinson's disease. Lancet Neuro. 2013;12:716-726.

41. Taubert M, Villringer A, Lehmann N. Endurance exercise as an "endogenous" neuro-enhancement strategy to facilitate motor learning. Front Hum Neurosci. 2015;9:692.

42. Harris TJ, Victor CR, Carey IM, Adams R, Cook DG. Less healthy, but more active: opposing selection biases when recruiting older people to a physical activity study through primary care. BMC Public Health. 2008;8:182.

43. de Souto Barreto P, Ferrandez AM, Saliba-Serre B. Are older adults who volunteer to participate in an exercise study fitter and healthier than nonvolunteers? The participation bias of the study population. $J$ Phys Act Health. 2013;10:359-367. 